\title{
RELAÇÃO ENTRE POTENCIAIS EVOCADOS AUDITIVOS DE MÉDIA LATÊNCIA E DISTÚRBIO DE PROCESSAMENTO AUDITIVO: ESTUDO DE CASOS
}

\author{
Relationship between auditory evoked potentials and middle \\ latency auditory processing disorder: cases study
}

\author{
Ana Carla Leite Romero (1), Bruna Balisa Sorci (2), Ana Cláudia Figueiredo Frizzo (3)
}

\begin{abstract}
RESUMO
O Potencial Evocado Auditivo de Média Latência é um teste objetivo promissor na audiologia na pesquisa neuro-diagnóstica das disfunções do sistema auditivo. Tem como vantagens a precisão e objetividade na avaliação e por isso é útil em crianças. O presente estudo teve como objetivo analisar os potenciais evocados auditivos de média latência em dois pacientes com distúrbio de processamento auditivo e relacionar as medidas objetivas e comportamentais. Para tanto foi realizado estudo de caso de dois pacientes ( $\mathrm{P} 1=$ feminino, 12 anos; $\mathrm{P} 2=$ masculino, 17 anos), ambos com ausência de alterações sensoriais, distúrbios neurológicos, neuropsiquiátricos. Ambos foram submetidos à anamnese, inspeção do meato acústico externo, avaliação audiológica e avaliação do exame de potencial evocado auditivo de média latência. Houve associação significante entre os resultados dos exames comportamentais e objetivos. Na anamnese, houve queixas referentes à dificuldade de escuta em ambiente ruidoso, localização sonora, desatenção, além de trocas fonológicas na escrita e na fala. Foram observadas alterações no processo de decodificação auditiva à direita em ambos os casos na avaliação comportamental do processamento auditivo e no exame de potencial evocado auditivo de média latência a resposta da via contralateral direita foi deficitária, confirmando as dificuldades dos pacientes estudados na atribuição de significado às informações acústicas em condição de competição sonora à direita nos dois casos. Para os casos estudados comprovou-se à associação entre os resultados, porém há necessidade de novos estudos com maior amostra para confirmação dos dados.
\end{abstract}

DESCRITORES: Percepção Auditiva; Potenciais Evocados Auditivos; Transtornos da Percepção Auditiva

(1) Fonoaudióloga; Mestranda em Fonoaudiologia pela Faculdade de Filosofia e Ciências da Universidade Estadual Paulista - FFC/UNESP, Marília,SP, Brasil; Membro dos grupos de pesquisa Avaliação da Linguagem e Fala e Avaliação e Intervenção dos Desvios da Linguagem, Fluência e Aprendizagem do Centro de Estudos da Educação e Saúde da Faculdade de Filosofia e Ciências - LIFAL/CEES/FFC/ UNESP, Marília, SP.

(2) Fonoaudióloga; Especialização em Audiologia Clínica pela Universidade de Ribeirão Preto - UNAERP, SP.

(3) Fonoaudióloga; Docente do Departamento de Fonoaudiologia e Programa de Pós-Graduação em Fonoaudiologia da Faculdade de Filosofia e Ciências da Universidade Estadual Paulista - FFC/UNESP, Marilia, SP, Brasil; Doutora em Neurologia pela Faculdade de Medicina de Ribeirão Preto - USP; Membro do grupo de pesquisa Avaliação da Linguagem e Fala.

Conflito de interesses: inexistente

\section{INTRODUÇÃO}

O Potencial Evocado Auditivo de Média Latência (PEAML) é um dos testes objetivos mais promissores na identificação de alteração de sistema nervoso auditivo central (SNAC). É composto por uma série de ondas que ocorrem entre 10 e 80 milissegundos (ms) após o início do estímulo auditivo. Caracterizado por ondas de pico negativo $(\mathrm{N})$ e de pico positivo $(P)$ é representado por uma sequência de letras alfabéticas como $\mathrm{PO}, \mathrm{Na}, \mathrm{Pa}, \mathrm{Na}, \mathrm{Pb}, \mathrm{Nb}$. $\mathrm{A}$ onda $\mathrm{Pa}$ é a mais consistente e frequentemente utilizada no diagnóstico, que pode ser comparável com a onda V do PEATE, devido sua amplitude mais facilmente visualizada. A primeira onda a ser 
registrada é a $\mathrm{Na}$, com latência em torno de 12 a $15 \mathrm{~ms}$, em seguida a $\mathrm{Pa}$, com $25 \mathrm{~ms}$, depois a $\mathrm{Nb}$ e $\mathrm{Pb}$. A análise da deflexão da $\mathrm{Na}$ é a mais importante, uma vez que seu traçado é mais constante ${ }^{1}$.

Os critérios de análise de resposta estão em função dos valores de latência (milissegundos-ms) e amplitude (microvolt-mv), sendo que a diminuição da intensidade provoca aumento dos valores de latência e diminuição nos valores de amplitude. A amplitude é o indicador para as alterações funcionais, pois os valores de latência apresentam muitas variações ${ }^{2}$.

Os PEAML representam uma importante ferramenta para a avaliação da função cerebral. Nos últimos anos, pesquisas vêm mostrando que esses potenciais relacionam-se aos núcleos e às vias auditivas situadas na região tálamo-cortical e córtex auditivo primário, principalmente no trato tálamo-cortical. A captação desses potenciais reflete às atividades corticais envolvidas nas habilidades auditivas primárias (reconhecimento, discriminação e figura-fundo) e não primárias (atenção seletiva, sequência auditiva e integração áudio-visual) ${ }^{3,4}$.

Os PEAML também podem ser utilizados para confirmar ou mesmo estudar estas condições clínicas relacionadas ao Distúrbio de Processamento Auditivo (DPA), auxiliando no neurodiagnóstico e colaborando no entendimento do SNAC. As principais vantagens do seu uso clínico são a precisão e objetividade, sendo que não são dependentes da resposta do paciente e podem ser de grande utilidade na avaliação de crianças com alteração no processamento auditivo (PA) e no monitoramento do processo terapêutico ${ }^{5}$.

Qualquer perda ou atraso que aconteça em algumas das etapas do PA origina o que se denomina DPA. O objetivo da avaliação de PA é determinar a presença ou ausência do distúrbio, descrever a extensão do mesmo, avaliar os efeitos da maturação do SNAC, detectar o 'local' da disfunção auditiva, definir as habilidades preferenciais para a aprendizagem, estabelecer a reabilitação e avaliar os benefícios de tratamento ou encaminhamento a outros profissionais ${ }^{6,7}$. A avaliação comportamental da função auditiva central é composta de testes especiais que observam o comportamento auditivo, como testes dicóticos, testes de processamento temporal, testes monoaurais de baixa redundância e testes binaurais ${ }^{8}$.

No Brasil, os testes mais utilizados são os dicóticos de dígitos, testes de escuta de dissílabos alternados (SSW), testes pediátricos com mensagem competitiva contralateral e ipsilateral (PSI-MCC e MCl) e testes de sentenças sintéticas com mensagem competitiva contralateral e ipsilateral (SSI-MCC e MCl). Testes de processamento temporal são de habilidades de reconhecimento e ordenação temporal de estímulos não verbais, os testes mais usados são os testes de padrões sequenciais de frequência e duração. Os testes monoaurais de baixa redundância avaliam o fechamento auditivo como nos testes de fala filtrada/ passa baixo e fala no ruído?.

Os tipos de prejuízos envolvidos no DPAC são quanto aos processos decodificação, codificação e organização. A dificuldade de decodificação se refere à inabilidade para atribuir significado à informação sensorial auditiva, como por exemplo, processo referente à análise do sistema fonêmico da linguagem. O prejuízo de codificação relaciona-se à inabilidade de integrar informações sensoriais auditivas e associá-las a outras informações sensoriais ou cognitivas. A dificuldade de organização, se refere à inabilidade de sequencializar eventos sonoros no tempo ${ }^{9}$.

Deste modo, o objetivo deste estudo foi analisar os potenciais evocados auditivos de média latência em dois pacientes com distúrbio de processamento auditivo e relacionar as medidas objetivas e comportamentais.

\section{APRESENTAÇÃO DOS CASOS}

O estudo de casos aqui apresentado é do tipo descritivo analítico.

Os casos foram identificados no estudo como: (1): Paciente 1 (P1): J., 12 anos de idade, 3. ${ }^{a}$ série do ensino fundamental, nascido a termo; (2): Paciente 2 (P2): A., 17 anos de idade, 3. ${ }^{a}$ série do ensino médio, nascido a termo.

Ambos foram submetidos à avaliação audiológica básica, exame do processamento auditivo central e teste do potencial evocado auditivo de média latência na Clínica Escola do Departamento de Fonoaudiologia da Universidade de Ribeirão Preto conforme autorização da responsável Profa Ms. Juliana Rocha. Os dois pacientes são atendidos na Clínica de Audiologia da instituição de origem.

Inicialmente os pacientes foram submetidos à anamnese, inspeção do condutivo auditivo, e avaliação audiológica, usando o audiômetro modelo AD-28, marca Interacoustics e analisador de orelha média AZ-7, também da marca Interacoustics, além de sistema portátil de captação de respostas evocadas, modelo Traveler Express, da marca Bio-logic System Corporation, para a medida do potencial evocado de média latência.

A avaliação simplificada do processamento auditivo foi iniciada com o teste do reflexo cócleo-palpebral com o instrumento agogô. No teste de localização sonora foi utilizado o instrumento guizo. 
Utilizou-se também o teste de memória sequencial não-verbal com os instrumentos sino, guizo, coco e agogô, sendo solicitado aos pacientes que apontassem a sequência destes instrumentos. Após este foi realizado o teste de memória sequencial verbal com as sílabas PA, TA, CA e FA, no qual os pacientes eram instruídos a repetir a sequência que fora apresentada. A bateria dos testes especiais foi iniciada com a pesquisa da fala com ruído, com apresentação de 40dBNS, o teste de fala filtrada foi apresentado em 50dBNS e com filtro passa-baixo; o teste de fusão binaural com apresentação em 40dBNS, na orelha sob teste em condição passa-baixo e na orelha contralateral em condição passa-alto. O teste de dicótico não-verbal foi realizado com 50dBNS acima da média em uma ordem de atenção livre, atenção à direita e à esquerda. $O$ teste dicótico de dígitos avaliou a integração binaural e a atenção direcionada à direita e à esquerda. A avaliação do teste SSW (teste de escuta de dissílabos alternados) foi com apresentação de 50 dBNS nas orelhas direita não-competitiva (DNC), direita competitiva (DC), esquerda não-competitiva (ENC) e esquerda competitiva (EC). Realizou-se o teste PSI em português (teste de escuta monótica e dicótica com sentenças), com apresentação de 40dBNS com mensagem competitiva contralateral (MCC) e mensagem competitiva ipsilateral (MCI), esta última com relação de -10, -15 dBNA.

Para o exame de potencial evocado auditivo os pacientes foram acomodados em poltrona reclinável e orientados a permanecerem despertos. A impedância elétrica esteve abaixo de 5 kohms e a diferença dos eletrodos foi de no máximo 3 kohms. Os eletrodos foram fixados na pele de forma que os ativos ficassem nas posições C3 e C4 em referência aos lóbulos da orelha direita (A2) e esquerda (A1), resultando na combinação C4/A2 e C3/A1, registrados ipsilateral (ai) e contralateral (ac), simultaneamente, utilizando os dois canais do equipamento seguindo o Padrão Internacional ${ }^{10}$.

Foram utilizados estímulos tone burst na modalidade monoaural, aplicado à intensidade de $70 \mathrm{~dB}$ NA de uma maneira aleatória, em velocidade de 10 estímulos/segundo, tempo de análise de $100 \mathrm{~ms}$, filtro passa-banda de 3 a $100 \mathrm{~Hz}$ polaridade alternada, sensibilidade de $75 \mu \mathrm{V}$.

$\mathrm{Na}$ análise deste teste, foram comparados os traçados obtidos de amplitude e latência das ondas $\mathrm{Na}$ e $\mathrm{Pa}$. A amplitude foi medida do pico negativo $\mathrm{Na}$ até o pico positivo $\mathrm{Pa}$. A medida de latência foi estabelecida no ponto de máxima deflexão da onda $\mathrm{Na}$ e $\mathrm{Pa}$. $\mathrm{O}$ efeito de orelha foi considerado quando na comparação de uma orelha à outra (OD e OE), a amplitude de uma orelha era significantemente menor que a outra (50\%), podendo ser ipsi ou contralateral ao local da lesão ou da disfunção. $\mathrm{O}$ efeito de eletrodo foi constatado quando na comparação da medida do hemisfério direito (C4) A2) com o esquerdo (C3/A1) a amplitude de um hemisfério era significantemente menor $(50 \%)$ que o outro hemisfério, podendo ser ipsi ou contralateral ao local da lesão ou da disfunção ${ }^{2}$.

O presente estudo teve a aprovação do Comitê de Ética da Universidade de Ribeirão Preto da UNAERP conforme protocolo n. $-183 / 07$. Trata-se de um estudo de caso clínico de dois pacientes atendidos na Clínica de Audiologia da Universidade de Ribeirão Preto - Unaerp.

\section{RESULTADOS}

$\mathrm{Na}$ anamnese ambos os casos apresentavam trocas fonoarticulatórias na fala e na escrita, dificuldade de compreender a conversação, dificuldade de localização sonora, demora no aprendizado da leitura e da escrita e história de otites nos primeiros anos de vida. $\mathrm{Na}$ atualidade, ambos pacientes apresentam bom estado de saúde geral, não fazem uso de medicação e realizam terapia fonoaudiológica no momento da avaliação na área de motricidade oral e linguagem oral e escrita.

Os limiares tonais aéreos estiveram na média de 20 dBNA para $\mathrm{P} 1$ e 05 dBNA para $\mathrm{P} 2$ nas frequências de 250 à $8000 \mathrm{~Hz}^{11}$ e as medidas de imitância acústica apresentaram curvas timpanométricas do tipo A, bilateralmente, em ambos os casos com reflexos acústicos contralaterais presentes ao nível médio de $100 \mathrm{~dB}$ para $\mathrm{P} 1$ e $\mathrm{P} 2^{12}$.

A Tabela 1 corresponde aos resultados da pesquisa de processamento auditivo. Observou-se no paciente 1 , testes monóticos de baixa redundância (ruído branco e de fala filtrada) alterados à orelha direita. No paciente 2 o teste dicótico de dissílabos alternados SSW esteve alterado à direita. As respostas obtidas no teste de processamento auditivo sugerem alteração do tipo decodificação de grau leve à direita no paciente 1 e do tipo decodificação de grau moderado à direita no paciente 2 .

Os valores apresentados na Tabela 2 correspondem às respostas do potencial de média latência dos pacientes em estudo. $\mathrm{O}$ componente $\mathrm{Pb}$ não foi identificado uma vez que faziam parte dos critérios de análise deste estudo apenas os componentes $\mathrm{Na}-\mathrm{Pa}-\mathrm{Nb}$. Estão presentes os valores de latência de cada onda e a relação de amplitude $\mathrm{Na}-\mathrm{Pa}$. 
Tabela 1 - Resultados da pesquisa de processamento auditivo

\begin{tabular}{|c|c|c|}
\hline TESTES REALIZADOS & RESULTADOS P1 & RESULTADOS P2 \\
\hline Reflexo cócleo-palpebral & Presente & Presente \\
\hline Hab. localização sonora & 5 acertos em 5 direções - normal & 5 acertos em 5 direções - normal \\
\hline $\begin{array}{l}\text { Hab.memória sequencial de } \\
\text { sons verbais }\end{array}$ & 2 acertos em 3 sílabas - normal & 3 acertos em 3 sílabas - normal \\
\hline $\begin{array}{l}\text { Hab.memória sequencial de } \\
\text { sons não verbais }\end{array}$ & $\begin{array}{l}3 \text { acertos em } 4 \text { instrumentos - } \\
\text { normal }\end{array}$ & $\begin{array}{l}3 \text { acertos em } 4 \text { instrumentos - } \\
\text { normal }\end{array}$ \\
\hline $\begin{array}{l}\text { Teste de fala com ruído } \\
\text { branco }\end{array}$ & $\begin{array}{l}\text { OD } 56 \% \text { OE } 80 \% \text { - alterado à } \\
\text { orelha direita } \\
\text { (decodificação - grau leve) }\end{array}$ & OD $72 \%$ OE $88 \%$ - normal \\
\hline Teste fala filtrada & $\begin{array}{l}\text { OD } 64 \% \text { OE } 76 \% \text { - alterado à } \\
\text { orelha direita } \\
\text { (decodificação - grau leve) }\end{array}$ & OD $88 \%$ OE $88 \%$ - normal \\
\hline Teste dicótico não verbal & $\begin{array}{l}\text { Atenção livre OD } 10 \text { OE } 14 \\
\text { Escuta direcionada à direita - } 23 \\
\text { acertos - normal }\end{array}$ & $\begin{array}{l}\text { Atenção livre OD } 16 \text { OE } 16 \\
\text { Escuta direcionada à direita - } 24 \\
\text { acertos - normal }\end{array}$ \\
\hline Teste dicótico de dígitos & $\begin{array}{l}\text { Integração Binaural OD 96\% e OE } \\
96 \% \text { - normal }\end{array}$ & $\begin{array}{l}\text { Integração Binaural OD } 100 \% \text { e } \\
\text { OE } 97,5 \% \text { - normal }\end{array}$ \\
\hline SSW & $\begin{array}{l}\text { Avaliação quantitativa: condição } \\
\text { DC - } 97 \% \text { EC - 92\% } \\
\text { Avaliação qualitativa: nenhum } \\
\text { efeito - normal }\end{array}$ & $\begin{array}{l}\text { Avaliação quantitativa: condição } \\
\text { DC - } 77,5 \% \text { EC - } 90 \% \\
\text { (decodificação - grau moderado) } \\
\text { Avaliação qualitativa: nenhum } \\
\text { efeito }\end{array}$ \\
\hline \multirow[t]{2}{*}{ PSI } & $\begin{array}{l}\text { Tarefa dicótica - } 100 \% \text { em ambas } \\
\text { as orelhas } \\
\text { Tarefa monótica: OD - 100\% nas } \\
\text { relações 0dB e -10dB e } 90 \% \text { na } \\
\text { relação }-15 \mathrm{~dB}\end{array}$ & $\begin{array}{l}\text { Tarefa dicótica - } 100 \% \text { em ambas } \\
\text { as orelhas } \\
\text { Tarefa monótica: OD - } 80 \% \text { nas } \\
\text { relações } 0 \mathrm{~dB} \text { e }-10 \mathrm{~dB} \text { e } 70 \% \text { na } \\
\text { relação }-15 \mathrm{~dB}\end{array}$ \\
\hline & $\begin{array}{l}\text { relações } 0 \mathrm{~dB}, 100 \% \text { em }-10 \mathrm{~dB} \text { e } \\
70 \% \text { na relação }-15 \mathrm{~dB} \text {. } \\
\text { normal }\end{array}$ & $\begin{array}{l}\text { OE }-80 \% \text { nas } \\
\text { relações } 0 \mathrm{~dB}, 70 \% \text { em }-10 \mathrm{~dB} \text { e } \\
70 \% \text { na relação }-15 \mathrm{~dB} \text {. } \\
\text { Resultados normal }\end{array}$ \\
\hline
\end{tabular}

LEGENDA: Hab.= habilidade; SSW = teste de escuta de dissílabos alternados; $\mathrm{PSI}$ = teste pediátrico com dissílabos alternados; OD= orelha direita; $\mathrm{OE}=$ orelha esquerdo; $\mathrm{DC}=$ direita competitiva; $\mathrm{EC}=$ esquerda competitiva.

Tabela 2 - Registro das latências de cada onda ( $\mathrm{Na}, \mathrm{Pa}, \mathrm{Nb}$ e $\mathrm{Pb}$ ) ipsilateral e contralateral e dos valores de inter-amplitudes $\mathrm{Na}-\mathrm{Pa}$

\begin{tabular}{|c|c|c|c|c|c|}
\hline & & \multicolumn{3}{|c|}{ LATÊNCIA } & \multirow{2}{*}{$\begin{array}{c}\text { AMPLITUDE } \\
\mathrm{Na}-\mathrm{Pa}\end{array}$} \\
\hline & & $\mathrm{Na}$ & $\mathrm{Pa}$ & $\mathrm{Nb}$ & \\
\hline OD P1 & C4A2 ai & $20,6 \mathrm{~ms}$ & $28,8 \mathrm{~ms}$ & $37,8 \mathrm{~ms}$ & $1,29 \mu \mathrm{V}$ \\
\hline OD P1 & C3A1 ac & $15,6 \mathrm{~ms}$ & $23,4 \mathrm{~ms}$ & $30,6 \mathrm{~ms}$ & $0,71 \mu \mathrm{V}^{*}$ \\
\hline OE P1 & C3A1 ai & $16,3 \mathrm{~ms}$ & $24,9 \mathrm{~ms}$ & $33,5 \mathrm{~ms}$ & $1,43 \mu \mathrm{V}^{* *}$ \\
\hline OE P1 & $\mathrm{C} 4 \mathrm{~A} 2 \mathrm{ac}$ & $16,3 \mathrm{~ms}$ & $26,9 \mathrm{~ms}$ & $35,8 \mathrm{~ms}$ & $1,06 \mu \mathrm{V}$ \\
\hline OD P2 & C4A2 ai & $23,4 \mathrm{~ms}$ & $37,0 \mathrm{~ms}$ & $46,0 \mathrm{~ms}$ & $0,40 \mu \mathrm{V}$ \\
\hline OD P2 & C3A1 ac & $25,1 \mathrm{~ms}$ & $37,0 \mathrm{~ms}$ & $45,0 \mathrm{~ms}$ & $0,25 \mu \mathrm{V}^{*}$ \\
\hline OE P2 & C3A1 ai & $31,5 \mathrm{~ms}$ & $40,7 \mathrm{~ms}$ & $33,5 \mathrm{~ms}$ & $0,45 \mu \mathrm{V}^{\star *}$ \\
\hline OE P2 & $\mathrm{C} 4 \mathrm{~A} 2 \mathrm{ac}$ & $15,7 \mathrm{~ms}$ & $37,1 \mathrm{~ms}$ & $45,0 \mathrm{~ms}$ & $0,42 \mu \mathrm{V}$ \\
\hline
\end{tabular}

LEGENDA: OD= orelha direita; OE= orelha esquerdo; P1 + paciente 1; P2 =paciente2; C4/A2 = hemisfério direito; C3/A1 = hemisfério esquerdo; ai = registro ipsilateral; $a c=$ registro contralateral; $\mathrm{ms}=$ milissegundos; $\mathrm{mV}=$ microvolts; ${ }^{*}=$ efeito de orelha: ${ }^{* *}=$ efeito de eletrodo. 
Os valores de amplitude $\mathrm{Na}-\mathrm{Pa}$ na OD C3/ A1 contraleteral sugerem anormalidade para 0 paciente $1(\mathrm{P} 1)$. Na comparação entre as medidas das orelhas direita e esquerda tem-se efeito de orelha $\left(^{*}\right)$. Além disso, na comparação entre os hemisférios direito (C4/A2) e esquerdo (C3/A1), as medidas do hemisfério esquerdo são $50 \%$ menores que aquelas do hemisfério direito, o que mostra efeito de eletrodo $\left(^{* \star}\right)$ também no paciente 1 (P1).

Com relação ao paciente 2 (P2) foram também observadas diferenças entre as orelhas direita e esquerda, efeito de orelha $\left({ }^{*}\right)$, e entre os hemisférios direito (C4/A2) e esquerdo (C3/A1), efeito de eletrodo $\left({ }^{* *}\right)$. Em ambos os casos a atividade elétrica observada em OD C3/A1 contralateral também foi menor em relação às outras medidas e mais especificamente no paciente 2 (P2) a latência absoluta da onda Pa também esteve alongada em todas as medidas.

\section{DISCUSSÃO}

Neste estudo, o uso associado dos testes comportamentais do PA e PEAML foi útil e auxiliou numa melhor investigação do funcionamento do sistema auditivo central dos dois casos estudados diagnosticados com Distúrbio de Processamento Auditivo (DPA).

Segundo a literatura ${ }^{9}$, pessoas com DPA têm dificuldade de escutar e compreender em ambientes de conversação, reverberantes ou barulhentos. Além disso, são indicados àqueles que apresentam dificuldades de identificação da fonte sonora e atenção. As alterações podem se manifestar de maneiras variáveis, entre estas estão: distração perda da capacidade de concentração; dificuldade para entender a fala na presença de ruído e dificuldade na expressão da fala e de aprendizagem na leitura e na escrita ${ }^{9}$ Estas dificuldades são mais evidenciadas em pacientes com alteração de decodificação da informação sonora ${ }^{13}$ e foram coincidentes com aquelas identificadas durante a anamese nos dois casos estudados.

Os dados da Tabela 1 mostram alterados os testes relacionados às habilidades de fechamento auditivo e os testes de fala com ruído branco e fala filtrada, mostrando alterações na orelha direita para o paciente P1. Já para o paciente P2, o teste SSW na análise quantitativa mostrou-se alterado nas condições de escuta competitiva à direita. Tais achados sugerem em ambos os casos dificuldade na recepção das informações sonoras, especialmente quando o teste é realizado com informações acústicas degradadas ou em condições de escuta difícil na orelha direita nos dois casos. A conclusão do exame indica uma alteração de processamento auditivo do tipo decodificação de grau leve no P1 e de grau moderado no $\mathrm{P}^{9}{ }^{9}$

Os valores de latência encontrados para $\mathrm{Na}, \mathrm{Pa}$ e Nb não tiveram diferenças muito discrepantes com o valor de normalidade para o paciente P1 (Tabela 2). A literatura da área ${ }^{14}$ sugere para a faixa etária média de 20,65 ms para $\mathrm{Na}$ e 29,81 ms para $\mathrm{Pa}$ e de 20,7 ms para $\mathrm{Na}$ e de 35,3 ms para $\mathrm{Pa}^{15}$. Já para o paciente P2 os valores de latência de Pa foram alongados na comparação dos valores obtidos na literatura ${ }^{16}$ para a mesma faixa etária com média de $17,91 \mathrm{~ms}$ para $\mathrm{Na}$ e de 29,41 para $\mathrm{Pa}$ e $20,77 \mathrm{~ms}$ para $\mathrm{Na} 31,07 \mathrm{~ms}$ para $\mathrm{Pa}^{17} \mathrm{em}$ relação aos valores $23 \mathrm{~ms}$ e $37 \mathrm{~ms}$ registrados no paciente $\mathrm{P} 2$.

A latência de Pa não deve ultrapassar $35 \mathrm{~ms}$ em pessoas sem lesões ou disfunções auditivas². $\mathrm{A}$ origem neural da onda $\mathrm{Pa}$ é atribuída à porção medial do giro de Heschl ${ }^{18,19}$ e as habilidades de reconhecimento e discriminação ao nível do córtex auditivo ${ }^{4}$ habilidades estas que estão prejudicadas no paciente $\mathrm{P} 2$.

$A$ atividade elétrica observada em OD C3/A1 contralateral foi sempre menor ${ }^{20}$ e produziu efeito de orelha em ambos os casos, sugerindo prejuízo na recepção acústica da via auditiva contralateral direita. A literatura ${ }^{20}$ tem relatado ainda que o efeito de orelha é sensível para evidenciar transtornos da via auditiva e do processamento da informação sonora.

Ainda no exame de PEAML, a análise das respostas registradas sobre o hemisfério esquerdo é bem menor quando comparada às outras medidas como mostra o efeito de eletrodo. Tal achado configura disfunção cortical auditiva e resulta em prejuízo nas habilidades de reconhecimento, discriminação e figura-fundo, entre outras e de associação da informação auditiva com informações linguísticas ${ }^{3,4}$.

Informações significantes as quais se referem à associação das habilidades auditivas alteradas no exame comportamental de PA com o exame de PEAML foram observadas neste estudo, o que confirma a efetividade do uso de medidas comportamentais e eletrofisiológicas para o diagnóstico e tratamento de indivíduos com DPA 5 .

A avaliação comportamental do PA evidenciou prejuízos no processo de decodificação auditiva, caracterizados pelas habilidades de fechamento auditivo prejudicadas e pela dificuldade do paciente na recepção das informações sonoras em condição de escuta difícil. $O$ exame de PEAML confirma tal prejuízo já que sua resposta mostra que a via contralateral direita e o hemisfério esquerdo são deficitários em ambos os casos.

A associação entre os resultados dos exames do processamento auditivo e do PEAML foi confirmada apenas para os casos estudados, o que sugere a 
necessidade de novos estudos com uma amostra maior para confirmação destes dados.

\section{CONCLUSÃO}

A análise comparativa dos resultados dos exames sugere associação das alterações do exame comportamental de PA e as alterações do exame de PEAML.

No exame comportamental foi caracterizada dificuldade quanto à recepção acústica da informação (prejuízo de decodificação à direita) em ambos os casos. O exame de PEAML confirma tal prejuízo já que a resposta da via contralateral direita e do hemisfério esquerdo são deficitárias e configuram disfunção de vias auditivas corticais.

$O$ exame de PEAML mostrou-se um instrumento útil no diagnóstico das disfunções da via auditiva para os casos estudados possibilitando a avaliação objetiva e associação com os testes comportamentais do PA o que justifica seu uso em pacientes com DPA.

\begin{abstract}
The Auditory Evoked Middle Latency Response is one of the most promising objective tests in audiology and in revealing brain dysfunction and neuro-audiologic findings. The main advantages of its clinical use are precision and objectivity in evaluating children. This study aimed to analyze the auditory evoked middle latency response in two patients with auditory processing disorder and relate objective and behavioral measures. This case study was conducted in 2 patients ( $P 1=12$ years, female, $\mathrm{P} 2=17$ years old, male), both with the absence of sensory abnormalities, neurological and neuropsychiatric disorders. Both were submitted to anamnesis, inspection of the external ear canal, hearing test and evaluation of Auditory Evoked Middle latency Response. There was a significant association between behavioral test and objectives results. In the interview, there were complaints about the difficulty in listening in a noisy environment, sound localization, inattention, and phonological changes in writing and speaking, as confirmed by evaluation of auditory processing and Auditory Evoked Middle Latency Response. Changes were observed in the right decoding process hearing in both cases on the behavioral assessment of auditory processing; auditory evoked potential test middle latency shows that the right contralateral via response was deficient, confirming the difficulties of the patients in the assignment of meaning in acoustic information in a competitive sound condition at right, in both cases. In these cases it was shown the association between the results, but there is a need for further studies with larger sample population to confirm the data.
\end{abstract}

KEYWORDS: Auditory Perception; Evoked Potentials, Auditory; Auditory Perceptual Disorders

\section{REFERÊNCIAS}

1. Geisler C, Frishkopf L, Rosenblith W. Extra cania Iresponses to acoustic clicks in man. Science. 1958; 128:1210-1.

2. Hall JW. New Handbook of auditory evoked responses. Allyn and Bacon: Massachusetts, 2006.

3. Tremblay KL. Training-related changes in the brain: evidence from human auditory-evoked potentials. Semin Hear. 2007; 28:120-32.

4. Kraus N, Mcgee TJ. The middle latency response generating system. Electroencephalogr Clin Neurophysiol. 1995; 44(suppl.); 93-101.

5. Schochat E, Musiek FE, Alonso R, Ogata J. Effect of auditory training on the middle latency response in children with (central) auditory processing disorder. Braz J Med Biol Res. August 2010; 43(8):777-85.
6. Rios AA, Rezende AG, Pela SM, Ortiz KZ, Pereira LD. Teste de padrão harmônico em escuta dicótica com dígitos - TDDH. Rev Soc Bras Fonoaudiol. 2007; 12(4):304-9.

7. Pinheiro FH, Oliveira AMO, Cardoso ACV, Capellini SA. Testes de escuta dicótica em escolares com distúrbio de aprendizagem. Braz J Otorhinolaryngol. 2010; 76(2):164-71.

8. Bellis TJ. Assessment and Management of Central Auditory Processing Disorders in the Educational Setting: from science to practice. San Diego: Singular Publishing Group, 1996. 349 p.

9. Pereira LD, Schochat E. Testes auditivos comportamentais para avaliação do processamento auditivo central. 1 ed. Carapicuiba: Pró-fono, 2011. 
10. Jasper HH. The ten twenty electrode system of the international federation. Electroencephalogr Clin Neurophysiol. 1958; 10:371-5

11. Davis $\mathrm{H}$, Silverman SR. Hearing and deafness. 4 ed. New York: Rinehardt \& Wilson, 1978.

12. Jerger J. Clinical experience with impedance audiometry. Arch Otolaryng. 1970;

92:311-24.

13. Meneguello J, Domenico MLD, Costa MCM, Leonhardt FD, Barbosa LHF, Pereira LD. Ocorrência de reflexo acústico alterado em desordens do processamento auditivo. Rev. Bras. Otorrinolaringol. 2001;67(6):830-5.

14. Costa SMB, Costa Filho AO, Cardoso MRA. Estudo do potencial evocado auditivo de média latência: efeito da orelha, sexo e idade. Pró-Fono. 2003; 15(2):181-8.

15. Frizzo ACF, Funayama CAR, Isaac $M L$, Colafêmina JF. Potenciais Evocados Auditivos de Média Latência: estudo em crianças saudáveis. Rev Bras Otorrinolaringol. 2007; 73(3):398-403. 16. Almeida FS, Pialarissi PR, Paiva Jr LEF, Almeida MAO, Silva A. Respostas auditivas evocadas de latência média: um estudo de padronização. Rev Bras Otorrinolaringol. 2006;72(2):227-34.

17. Neves IF, Gonçalves IC, Leite RA, Magliaro FCL, Matas CG. Estudo das latências e amplitudes dos potenciais evocados auditivos de média latência em indivíduos audiologicamente normais. Rev Bras Otorrinolaringol. 2007;73(1):75-80.

18. Yvert B, Crouzeix A, Bertrand $O$, Seither-Preisler A, Pantev C. Multiple supratemporal sources of magnetic and electric auditory evoked middle latency components in humans. Cereb Cortex. 2001;11:411-23.

19. Yvert B, Fischer C, Guenot M, Krolak-Salmon P, 2004; 70, Isnard J, Pernier J. Simultaneous intracerebral EEG recordings of early auditory thalamic and cortical activity in human. Europ $\mathrm{J}$ Neurosci. 2002;16:1146-50.

20. Schochat E, Rabelo CM, Loreti RCA. Sensitividade do potencial evocado auditivo de média latência. Rev Bras Otorrinolaringol, 2004; 70 (3):353-8.

Endereço para correspondência: Ana Cláudia Figueiredo Frizzo Avenida Hygino Muzzy Filho, 737 Marília - SP CEP: $17525-900$

E-mail: anafrizzo@marilia.unesp.br 